\title{
MECANISMO DE ACCIÓN DEL LEVONORGESTREL COMO ANTICONCEPTIVO DE EMERGENCIA*
}

\author{
Fernando Zegers Hochschild
}

La polémica que ha generado el uso del Levonorgestrel (como anticonceptivo de emergencia), se advierte en este artículo, obedece a la creencia de que si ya existe un óvulo fecundado, este fármaco puede impedir el desarrollo del embrión antes de que llegue al útero y/o alterar de tal manera el endometrio que impida su implantación en él. El autor señala que, aun cuando no existen estudios in vitro que evalúen el efecto del Levonorgestrel (LNG) en el desarrollo pre implantación de embriones humanos, dos hechos hacen suponer que no afecta el devenir del embrión. 1) Se sabe que el LNG, a diferencia de las antiprogestinas, no produce aborto o interrupción de un embarazo clínico cuando el embrión ya implantado se expone a este fármaco. Y 2), considerando que la mayor parte de los embarazos ocurren cuando el LNG es usado después de las 72 horas del coito, y

Fernando Zegers Hochschild. Médico cirujano. Director de la Unidad de Medicina Reproductiva, Clínica Las Condes, Santiago. Miembro del consejo de directores del Instituto Chileno de Medicina Reproductiva (ICMER). Presidente del Comité de Ética de la Sociedad Chilena de Fertilidad. Miembro del panel de expertos en reproducción humana de la Organización Mundial de la Salud. Fundador y miembro del directorio del Comité Internacional Responsable del Registro Mundial de la Reproducción Asistida (ICMART). Autor de numerosas publicaciones científicas.

* Ponencia presentada en el seminario "Los efectos de la píldora del día después" que se realizó en el Centro de Estudios Públicos el 2 de junio de 2004. Véanse asimismo en esta edición la ponencia del doctor Enrique Oyarzún, los artículos de los profesores de derecho Antonio Bascuñán y Arturo Fermandois, y el dossier "Anticoncepción de Emergencia: Antecedentes del Debate" (N. del E.). 
que habitualmente esto coincide con fases post ovulatorias, es razonable concluir que en la mayoría de los embarazos con LNG, el embrión se ha expuesto a él durante su fase pre implantacional. Por consiguiente, no existen evidencias de que los embarazos que se producen como falla del método anticonceptivo evolucionen en forma diferente de los embarazos que no estuvieron expuestos al LNG.

\section{CONSIDERACIONES GENERALES}

\subsection{Ciclo menstrual y período fértil}

$\mathrm{E}$ ciclo menstrual de la mujer se extiende desde el inicio de la menstruación (llamado día uno) hasta un día antes del inicio de la menstruación siguiente. El ciclo menstrual normal tiene una duración de 25 a 35 días. Por otra parte, el "período fértil" de la mujer corresponde a los días del ciclo menstrual en que una relación sexual coital puede dar inicio a un embarazo. En algunas mujeres, este período puede durar hasta seis días, desde cinco días antes de la ovulación (salida del óvulo del ovario) hasta el día de la ovulación propiamente tal. Sin embargo, en otras mujeres este período puede ser más corto y extenderse por sólo uno o dos días antes de la ovulación, restringiendo así el período fértil a sólo dos a tres días en el mes. Estas diferencias son determinantes en la mayor o menor capacidad para embarazarse de algunas mujeres respecto de otras.

En ocasiones, el período fértil puede ser identificado por la mujer mediante la humedad vaginal, que resulta de la producción de moco por el cuello del útero y que en algunas mujeres es abundante y baña la vagina. Este moco es el que posibilita la entrada de los espermatozoides hacia el útero después que han sido depositados en la vagina durante el coito. En ausencia de este moco cervical, los espermatozoides depositados en la vagina durante la relación sexual no pueden entrar al interior del útero y mueren rápidamente en la vagina.

Con frecuencia, las mujeres no pueden precisar si están en el inicio o en el fin del período fértil; más aún, rara vez las mujeres tienen conciencia del día del ciclo menstrual en que se encuentran al momento de tener un acto sexual no programado. Esto es especialmente evidente en adolescentes que es frecuente que tengan ciclos menstruales irregulares o no estén educadas para tomar conciencia de estos cambios fisiológicos.

Considerando que la mujer tiene relaciones sexuales dentro y fuera de su período fértil, por una cuestión de probabilidades la mayor parte de las relaciones sexuales $(80 \%)$ tienen lugar fuera del período fértil. Así, la 
FIGURA 1: $\quad$ PERÍODO FÉRTIL DE LA MUJER

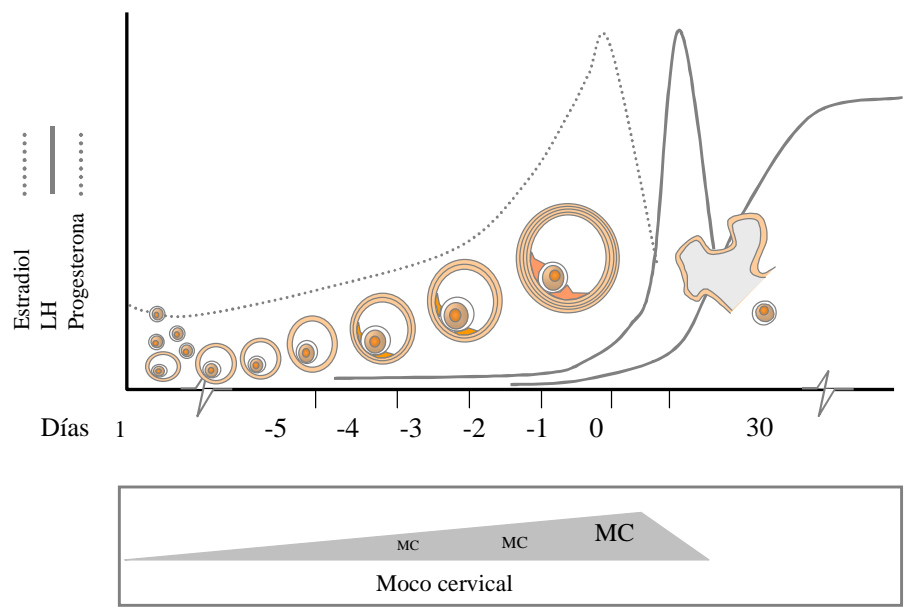

mayoría de las veces, lo que motiva el uso de un anticonceptivo de emergencia (AE) es el temor a una preñez no deseada y no la certeza de haber tenido relaciones sexuales en el período fértil. Desde otra perspectiva, asumiendo que la mujer reconoce su período fértil y toma el AE exclusivamente durante ese período, 75 a $85 \%$ de las veces lo habrá ingerido antes de la ovulación, es decir, antes de que exista un óvulo, y con mayor razón antes de que el óvulo haya sido fecundado. Si la mujer lo toma motivada exclusivamente por el susto y en completo desconocimiento del momento del ciclo en que se encuentra, tiene un $96,6 \%$ de probabilidades de tomarlo fuera del período del ciclo en que puede existir un óvulo fecundado (Figura 1).

\subsection{El ciclo ovulatorio}

En el ciclo menstrual de la mujer se reconocen diferentes estadios que corresponden a sucesivas fases de la actividad cíclica del ovario (Figura 2). En general se distingue la fase folicular (FF) o preovulatoria, que se extiende desde el inicio de la menstruación hasta la ovulación, y la fase lútea (FL), que se extiende desde la ovulación hasta el inicio de la menstruación siguiente. A su vez en la FF se pueden identificar tres subperíodos: 
FIGURA 2:

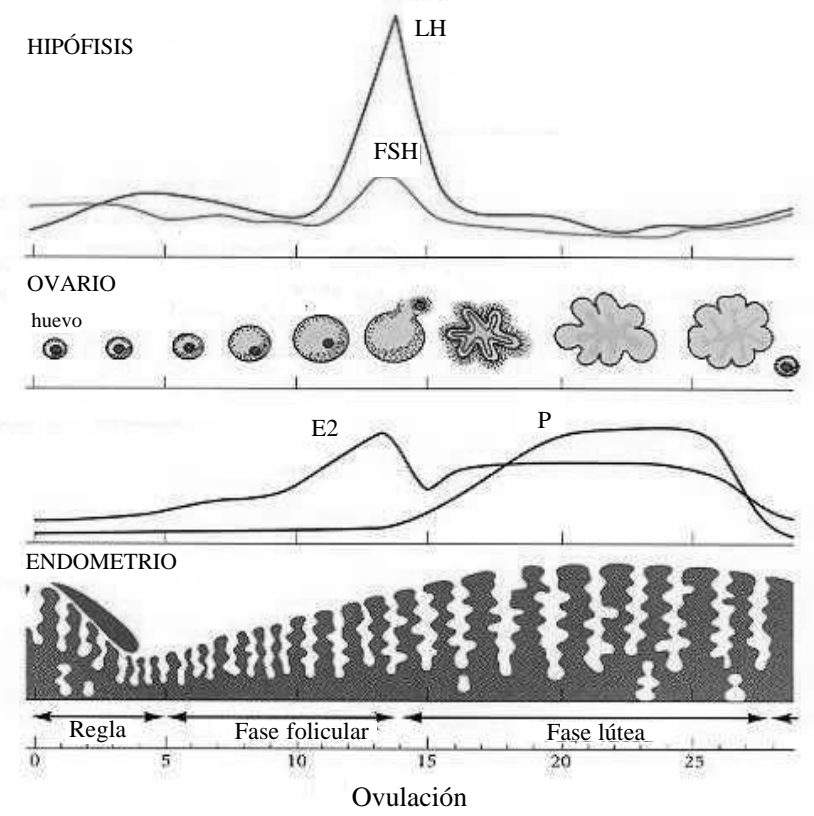

1) Reclutamiento folicular: en este período, gracias al estímulo de hormonas (LH y FSH) que produce y secreta la hipófisis (una glándula situada en la base del cerebro que controla la actividad cíclica del ovario), se seleccionan 10 a 12 folículos en uno o ambos ovarios (cohorte). Cada folículo contiene un óvulo que se prepara para la ovulación de ese ciclo. Esta fase se extiende desde la menstruación hasta aproximadamente el día 10 del ciclo.

2) Maduración folicular: en este período se selecciona sólo uno (ocasionalmente dos) de los folículos que habían sido reclutados en la fase anterior. Los otros folículos se reabsorben en un proceso llamado de atresia folicular. Al folículo seleccionado se le denomina folículo dominante, porque contiene el óvulo escogido para ovular en ese mes. El folículo dominante crece hasta un diámetro promedio de 16 a $24 \mathrm{~mm}$ antes de la ovulación. Esta fase se extiende hasta que la hipófisis secreta una mayor cantidad de LH, llamado pico de LH y que marca el inicio de la ovulación.

3) Ovulación: consiste en la ruptura de la pared del folículo dominante que permite la liberación del óvulo. Ésta es una etapa en que la 
cronología de eventos resulta crítica. Así, la hipófisis, que ha estado comandando el reclutamiento y maduración folicular mediante señales químicas (consistentes en pulsos hormonales de amplitud y frecuencia variables, de acuerdo al momento del ciclo), libera en la sangre una descarga hormonal llamada pico de LH. Eso determina que el óvulo salga del ovario en las siguientes 24 a 48 horas y, una vez capturado por la trompa, debe ser fecundado en las siguientes 12 a 24 horas. De no ser así, envejece y/o muere.

Si se suprimen los pulsos hormonales de la hipófisis, no se produce reclutamiento y maduración folicular. Los anticonceptivos hormonales de uso regular y que se ingieren todos los días impiden la secreción de estos pulsos hormonales y por ello suprimen la ovulación. Si ya han ocurrido el reclutamiento y la maduración folicular y se inhibe el pico de $\mathrm{LH}$, el folículo reclutado y madurado no se rompe y no libera el óvulo. Ocasionalmente se ha visto que, aun inhibiendo el pico hormonal, se puede romper el folículo en lo que podría llamarse una seudoovulación. Esto no significa que de la pared folicular se libere el óvulo o que, de ser liberado, el óvulo pueda ser fecundado. En ausencia del pico de LH el óvulo no es liberado de la pared folicular o no completa su maduración y, por lo tanto, es altamente improbable que un óvulo así generado sea fecundable. Esto es importante recordar, ya que, como veremos más adelante, uno de los mecanismos de acción del AE consiste en alterar la magnitud y la frecuencia de las señales hormonales provenientes de la hipófisis.

El ciclo ovulatorio es altamente controlado y para que ocurra una gestación se requiere no sólo de la producción de un óvulo fecundable; se requiere además que, sincrónicamente con lo anterior, se prepare el endometrio para la implantación de un embrión. En la fase folicular del ciclo ovárico, la cohorte de folículos reclutados y luego el folículo dominante producen una de las hormonas femeninas llamada estradiol (E2), que por una parte induce a las glándulas del cuello uterino a producir moco y por otra parte induce la proliferación y secreción de las glándulas endometriales o capa interna del útero. La mayor proliferación glandular ocurre al momento de la ovulación, coincidiendo con la mayor producción de E2, que precede en 24 horas al pico de LH. Así, esta hormona es la responsable de la proliferación endometrial que constituye el nido donde eventualmente se implantará un embrión.

El pico de LH, que ocurre a mediados del ciclo, no sólo provoca la ruptura del folículo, también esta señal es responsable de transformar las células del folículo e inducir un cambio en la producción hormonal que hace disminuir la producción de E2 y se inicia la producción de progestero- 
na (P). Es con la producción de esta nueva hormona que se da inicio a la fase lútea o posovulatoria. La $\mathrm{P}$ ejerce múltiples cambios dentro del ovario, así como en órganos distantes, los que pueden resumirse en: 1) es responsable de que el óvulo complete su maduración cromosómica (reinicie su meiosis) y se libere de la pared folicular; 2) es responsable de que las células que rodean al óvulo (células del cúmulo) se expandan y faciliten la posterior migración de espermatozoides a través de ellas; 3) a distancia, la $\mathrm{P}$ inhibe la producción de moco en el cuello del útero y con ello su aparición marca el cierre del período fértil; 4) también a distancia, la $\mathrm{P}$ transforma o madura las glándulas del endometrio, preparándolo para una eventual implantación embrionaria. La P es además la hormona responsable de mantener la preñez en las siguientes cinco semanas de gestación.

Es fácil comprender que cualquier intervención hormonal que tenga como resultado modificar el pico de LH modificará también la producción de $\mathrm{P}$, generando una innumerable secuencia de cambios que resultan en una severa alteración en la calidad de la ovulación. También es necesario comprender que muchos de los cambios inducidos en el nivel endometrial no se dan como hechos aislados, sino que en asociación a efectos generados en el óvulo o en la ovulación propiamente tal.

\subsection{Fecundación, transporte embrionario e implantación}

Una vez liberado del ovario, el óvulo debe ser captado por la trompa de Falopio dentro de las 24 horas de la ruptura folicular. En ese órgano es donde el óvulo completa su maduración y es en el fluido de la trompa donde ocurre la fecundación y donde vive el embrión por aproximadamente 72 horas. El embrión es entonces transportado al útero, donde continúa navegando en su fluido. A pesar de una activa interacción e intercambio de sustancias entre el embrión y la mucosa tubárica y endometrial, el embrión no parece tomar contacto directo con el endometrio hasta tres días después, en que se pone en marcha la adhesión al endometrio materno. A este proceso de aposición y luego invasión se le denomina implantación. Es necesario considerar que, con el conocimiento científico y tecnológico actual, no existe manera de reconocer marcadores químicos en la sangre de la mujer que identifiquen la presencia del embrión mientras éste viaja por la trompa de Falopio o navega en el fluido uterino (Figura 3).

El embarazo, algo que le pasa a la mujer y no al embrión, se establece desde que el embrión se implanta en el útero, ya que previo a ello no existe manera de saber si la mujer lleva o no un embrión en su interior. La manera más precoz de saberlo es a través de la medición, en la sangre de la 
FIGURA 3: $\quad$ ETAPAS TEMPRANAS DEL PROCESO REPRODUCTIVO DE LA MUJER

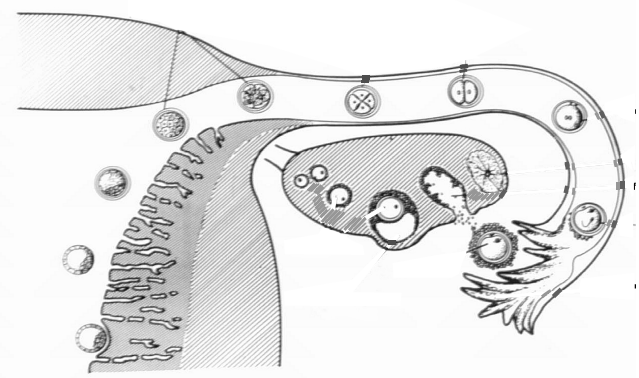

mujer, de una hormona producida exclusivamente por tejido embrionario llamada gonadotropina coriónica (hCG). Esa hormona se reconoce en la sangre de la mujer sólo después que el embrión se ha implantado en su endometrio, lo ha invadido y ha enviado esta señal hormonal por la sangre materna al ovario de la mujer, es decir después de seis días de la fecundación.

Al tomar conocimiento de que hay un embrión, el ovario mantiene la producción de $\mathrm{P}$ y con esto evita que llegue la menstruación siguiente. Esta relación temporal tiene relevancia semántica. No se puede hablar de gestación antes de que existan al menos señales químicas que documenten la presencia de un embrión en el cuerpo de la mujer. Considerando que aborto es la interrupción voluntaria o involuntaria de una gestación, no puede hablarse de aborto antes de tener evidencias de que existe un embrión. Esta definición no tiene que ver con el momento en la biología del desarrollo en que se constituye una nueva vida, sino más bien desde cuándo sabemos que esa nueva vida está presente.

\subsection{Fecundidad y eficiencia reproductiva}

Por fecundidad se entiende el tiempo que demora una mujer sana en embarazarse espontáneamente si tiene relaciones sexuales durante el período fértil con un hombre sano.

El tiempo que toma a una mujer embarazarse es mayor que en otros mamíferos, a pesar de que la mujer tiene períodos fértiles prácticamente todos los meses. Sin duda, cohabitar heterosexualmente por largos perío- 
dos ayuda a los propósitos reproductivos, ya que si bien la mujer acepta al hombre (al menos no lo rechaza) fuera de su período fértil, tampoco lo atrae específicamente durante esos períodos. En el mejor de los casos, la pareja cuenta con sólo 6 días al mes para que una relación sexual pueda generar un embarazo.

De 100 parejas sanas, menores de 35 años, probadamente fértiles y que tienen relaciones sexuales varias veces durante el período fértil, sólo 30 de ellas se embarazan clínicamente (con evidencia ecográfica de un saco gestacional intrauterino) en el primer mes de exposición (ZegersHochschild y cols., 1992). Lo interesante es preguntarse por qué el $70 \%$ restante no se embarazó, en circunstancias en que las mujeres ovularon bien y tuvieron relaciones sexuales efectivas durante el período fértil. Una de las hipótesis sería que algunas de ellas sí se embarazaron pero tuvieron pérdidas embrionarias ocultas, es decir, estuvieron embarazadas sin conocimiento de que ello había ocurrido. La respuesta se encuentra midiendo retrospectivamente la hormona hCG en el plasma congelado de las mujeres que no tuvieron evidencia clínica de embarazo. Así se pudo demostrar que de 100 parejas normales que tienen relaciones sexuales durante el período fértil, 68 mujeres tendrán evidencias químicas de haber tenido un embrión implantado. Sin embargo, sólo en 30 de ellas el embarazo seguirá adelante. Es decir, más de la mitad de los embriones que han logrado implantarse se pierden antes de haber evidencia clínica de su existencia, y pasan desapercibidos para la propia mujer. A este número deben sumarse los embriones que nunca llegaron a implantarse y de los que no se tiene evidencia directa, y deben sumarse además los embriones que se pierden después de establecido el embarazo clínico y que constituyen un $12 \%$ de todas las gestaciones en mujeres jóvenes. La primera enseñanza que deja este estudio es que la aparente ineficiencia reproductiva en nuestra especie es el resultado de una alta incidencia de pérdidas embrionarias ocultas llamadas también abortos subclínicos o bioquímicos. A éstos deben agregarse otro $12 \%$ de pérdidas embrionarias más tardías y que son reconocidas como abortos clínicos.

\section{Por qué se pierden tantos embriones}

Estudios efectuados en ovocitos humanos recuperados de mujeres sanas (Battaglia y cols., 1996) demuestran que ya en mujeres menores de 25 años, el 19\% de los ovocitos que se han seleccionado espontáneamente para ovular contienen alteraciones en el citoesqueleto y/o en el huso meiótico (donde se ordenan los cromosomas) que determinarán alteraciones en 
los embriones resultantes de la fecundación de esos óvulos. Esta proporción de óvulos anormales aumenta a 79\% en mujeres mayores de 40 años.

Nuestro grupo ha contribuido con estudios cromosómicos realizados en ovocitos recuperados de mujeres que participan en programas de reproducción asistida (Munné y cols., 2000). Como parte del proceso de maduración, y una vez liberados o extraídos del ovario, los óvulos deben eliminar la mitad de sus cromosomas antes de aceptar la otra mitad que aporta el espermatozoide. Así, se mantiene una dotación completa de cromosomas (46), al igual que en todas las células del organismo. El óvulo elimina la mitad de los cromosomas, empaquetados en una estructura llamada polocito o corpúsculo polar. Hemos desarrollado una técnica que permite obtener los cromosomas presentes en el polocito y que representan la imagen en espejo de los cromosomas que quedan dentro del óvulo antes de exponerlos al espermatozoide. Al examinar tan sólo 6 de los 23 cromosomas presentes en el polocito del óvulo maduro, se observa que la frecuencia de óvulos con alteraciones cromosómicas numéricas es altísima y aumenta con la edad de la mujer (Crosby y cols., 2004) (Cuadro 1).

\begin{tabular}{lccc}
\hline & $<35$ años (\%) & 35-39 años (\%) & $\geq 40$ años (\%) \\
\hline $\mathrm{N}^{\circ}$ de mujeres & 27 & 14 & 8 \\
$\mathrm{~N}^{\circ}$ de biopsias & 138 & 126 & 42 \\
$\mathrm{~N}^{\circ}$ de CP examinados & 109 & 111 & 34 \\
$\mathrm{~N}^{\circ}$ de aneuploidías (\%) & $26(23,9 \%)$ & $34(30,6 \%)^{*}$ & $23(67,6 \%) *$ \\
\hline
\end{tabular}

$* \mathrm{P}<0,0001$.

Fuente: Crosby y cols.: "El Corpúsculo Polar: Implicancias Éticas", 2004.

Lo que se aprende de estos estudios es que la eficiencia o ineficiencia reproductiva en nuestra especie está en gran medida determinada por la calidad de los óvulos que la mujer produce espontáneamente o luego de estimulación hormonal de la ovulación. Estos óvulos cromosómicamente anormales no impiden la fecundación pero producen embriones cromosómicamente anormales. Hoy se sabe que el $70 \%$ de los embriones producidos en mujeres de 36 años o más tienen alteraciones en el número de cromosomas (aneuploidías) que impiden su implantación o permanecen 
implantados por cortos períodos (Gianaroli et al., 2000). Por este motivo, la mayoría de los embriones generados en la naturaleza no se implantan o se pierden rápidamente después de la implantación. Una minoría sigue adelante y se pierde tardíamente. Un ejemplo de alteración cromosómica que logra seguir adelante, incluso hasta el nacimiento, lo constituye la trisomía del cromosoma 21 o "síndrome de Down". El análisis cromosómico practicado en 609 abortos espontáneos ocurridos en la Clínica Las Condes demuestra que el $67 \%$ se debe a alteraciones numéricas en los cromosomas (Be et al., 1997). Todas estas evidencias científicas confirman que las alteraciones cromosómicas presentes en los embriones - y que son parte de la matemática con que opera nuestra naturaleza- explican la mayoría de los abortos clínicos y subclínicos u ocultos.

Desde una perspectiva diferente, Wilcox y cols. $(1995$; 1998) estudiaron la probabilidad de embarazo en mujeres jóvenes y sanas que tenían sólo una relación sexual en el período fértil. Las mujeres que participaron en estos estudios llevaban registro de los días en que tenían relaciones sexuales y determinaban además el día de la ovulación midiendo hormonas en orina. Al igual que en los estudios señalados anteriormente, la eficiencia reproductiva aun en parejas probadamente fértiles es muy baja; y la frecuencia de pérdidas embrionarias ocultas es muy elevada (Cuadro 2). Es sorprendente que si una pareja sana tiene una relación sexual solamente en el día de la ovulación, la probabilidad de embarazo clínico no supera un $8 \%$ y la de tener un aborto subclínico excede el $50 \%$.

De éste y otros estudios de fecundidad se concluye que: 1) la mujer es fértil sólo seis días en el mes; 2) de esos seis días, cinco ocurren antes de que haya un óvulo fecundable en la trompa y, por cierto, antes de que 
exista un embrión; 3) una relación sexual el día de la ovulación tiene una probabilidad natural de concepción de alrededor de 38\%, sin embargo la probabilidad de embarazo clínico es tan sólo un $8 \%$, el resto se pierde en forma oculta.

\subsection{Eficiencia del LNG como anticonceptivo de emergencia}

La eficiencia del LNG (Levonorgestrel), usado como AE, se ha calculado comparando el número de embarazos resultantes en mujeres que lo habían usado después de un coito no protegido, con el número de embarazos que se hubieran producido si no lo hubieran usado. Para saber cuántos embarazos se debieran haber producido, se usa como referencia de embarazos esperados los resultados que se muestran en el Cuadro 2. Sin embargo, al usar esta comparación no se puede obtener un cálculo real de la eficacia porque los resultados, es decir, los embarazos obtenidos o prevenidos con el AE, no se calculan del mismo modo. La probabilidad de embarazos en el grupo de referencia (los esperados) se obtuvo en parejas sanas y reconocidamente fértiles, en las cuales se pudo definir claramente el día en que tuvieron la relación sexual con respecto del día de la ovulación. En cambio en el grupo de mujeres que reciben LNG como AE (los prevenidos), el día de la ovulación se estima preguntándoles a las mujeres cuándo había sido la última regla y asumiendo por la longitud de los ciclos si el día de la relación sexual coincide o no con alguno de los días del período fértil. Es decir, los estudios de eficiencia del LNG asumen mediante cálculos muy imprecisos si las mujeres expuestas estaban o no en el período fértil al tener relaciones sexuales.

Además es importante considerar que a diferencia del grupo que se usa como referencia, las mujeres que solicitan el AE tienen: 1) una fecundidad desconocida y es razonable suponer que al menos un 8 a $12 \%$ de ellas son infértiles; 2) que la inmensa mayoría de ellas no saben si estaban o no en el período fértil al tener la relación sexual no protegida, y por lo tanto una gran proporción de mujeres toma el AE sin necesitarlo. La mayoría de las mujeres no saben cuándo ovulan y a menudo no recuerdan el día exacto de su última menstruación. De tal manera que el cálculo del riesgo de embarazo establecido mediante el interrogatorio a mujeres estresadas por el susto de un embarazo no deseado, es poco preciso y está lleno de errores (Espinos y cols., 1999); 3) las mujeres que solicitan AE no quieren embarazarse. De hecho, muchas de ellas tuvieron coitos interrumpidos y, en otros casos, ruptura o desplazamiento de un condón que en la mayoría de los casos posee una sustancia llamada nonoxinol, que mata los esperma- 
tozoides. Existen varios estudios que demuestran que en más del $40 \%$ de estos casos no se registra la presencia de espermatozoides móviles en el cuello uterino al momento de solicitar el AE (Senosiain y cols., 2001); es decir, son relaciones sexuales que de ninguna manera hubieran producido un embarazo. Si bien la eficiencia anticonceptiva publicada fluctúa entre 70 y $85 \%$ (Von Hertzen y cols., WHO, 2002), las consideraciones antes mencionadas son relevantes a la hora de medir la efectividad de los anticonceptivos de emergencia. Análisis detallados de estas consideraciones sugieren que la eficiencia de los $\mathrm{AE}$ es baja en comparación con otros métodos hormonales de uso regular, como los anticonceptivos que se toman diariamente y cuya eficiencia es de aproximadamente 99,6\%. Es por esta eficiencia mucho menor que el LNG debe ser considerado sólo para uso de emergencia cuando otras formas de prevención del embarazo han fallado.

\section{MECANISMO DE ACCIÓN DEL LEVONORGESTREL COMO AE}

Antes de examinar los mecanismos de acción del Levonorgestrel (LNG), debe quedar claro que esta sustancia química es una progestina sintética que, al igual que otras de la misma familia, tiene acciones similares a la progesterona (P). Si se administra antes de la ovulación, al igual que la $\mathrm{P}$ natural, actúa como un antiestrógeno en el nivel periférico (inhibe las glándulas del endometrio y las glándulas del cuello uterino) y, en el nivel central, inhibe las descargas hormonales de la hipófisis. Si se administra después de la ovulación, debido a su gran afinidad con las glándulas endometriales, potencia el efecto de la $\mathrm{P}$ natural. Es por este efecto que por muchos años otras progestinas, como la medroxiprogesterona, se han usado en forma empírica, desde la ovulación en adelante, para facilitar la implantación embrionaria y evitar el aborto en mujeres que presentan abortos espontáneos repetidos. No existen evidencias científicas de que estos tratamientos tengan utilidad clínica; sin embargo, su uso por vía oral e inyectable es práctica frecuente entre ginecólogos hasta la actualidad. Por ello, si alguien quisiera diseñar un agente abortivo que impidiera la implantación embrionaria o alterara la arquitectura endometrial, no elegiría un agente progestacional como las progestinas sintéticas.

A continuación examinaré el mecanismo de acción del AE usado en diferentes momentos del ciclo de la mujer. Se entenderá por AE la sustancia que contiene $\mathrm{LNG}$ en dosis de $0,75 \mathrm{mg}$ a ser usado por dos veces con un intervalo de 12 horas. 


\subsection{LNG durante la fase folicular}

Si el LNG se administra en la "fase de reclutamiento folicular" ocurren fundamentalmente dos cosas:

1. Se retrasa la fase de reclutamiento folicular, retrasando proporcionalmente el momento de la ovulación o impidiendo que ésta ocurra.

2. El folículo elegido como dominante se reabsorbe en un proceso llamado atresia folicular. Esto puede ocasionar un pequeño sangrado que es el reflejo de que el endometrio perdió sustento hormonal.

Que ocurra lo uno o lo otro depende del momento en el desarrollo folicular en que se administra el medicamento y también de diferencias entre una persona y otra.

Si el LNG se administra en la "fase de maduración folicular", cercana a la ovulación, el efecto progestativo del LNG puede inhibir el crecimiento y maduración folicular, con lo que habitualmente se desencadena una atresia folicular. A diferencia del período anterior, lo habitual es que la ovulación se inhiba (aunque puede postergarse). También puede ocurrir un pequeño sangrado uterino semejante a una menstruación.

En una reciente investigación (Durand y cols., 2001), se administró LNG el día 10 del ciclo menstrual que corresponde a la fase inicial o media de maduración folicular. En 12 de 15 mujeres hubo inhibición de la ovulación (no hubo ruptura folicular ni fase lútea) y en otras tres mujeres se postergó la ovulación más allá del límite máximo de 6 días. El LNG administrado en la fase folicular tardía tiene la capacidad de interferir con el proceso ovulatorio, ya sea suprimiendo el pico de $\mathrm{LH}$, la ruptura folicular o la luteinización. Hay otros componentes del proceso ovulatorio muy importantes, como la maduración somática y germinativa del óvulo (reactivación de la meiosis y eliminación de la mitad de la dotación cromosómica), que son dependientes del tiempo de exposición al LH (horas después del pico de LH) y de la cantidad de LH secretada por la hipófisis. Esto significa que aunque existan evidencias ecográficas de ovulación (ruptura folicular), si se ha inhibido el pico de LH lo más probable es que, de liberarse el óvulo, éste no sea fecundable.

Por ser el AE una progestina, su administración en la fase folicular, además, altera el moco cervical, impidiendo, retrasando o dificultando la migración espermática. Aquellos espermatozoides que, producto de un coito previo, ya estaban a la espera del óvulo en el cuello uterino o en la trompa de Falopio, se encuentran con la alteración de los fluidos de estos órganos, producida por el AE, lo que dificulta o impide su migración, y por consiguiente el encuentro con el óvulo y la fecundación (Kesseru et al., 1973). 
Las investigaciones realizadas en este período permiten certificar que el $\mathrm{AE}$ administrado en la fase de reclutamiento y maduración folicular altera el ciclo ovulatorio, inhibiendo o postergando la ovulación y alterando la capacidad migratoria y fecundante de los espermatozoides.

\subsection{LNG durante el período ovulatorio}

La ovulación normal es el proceso mediante el cual el ovario libera un óvulo fecundable. Para que esto ocurra, la hipófisis produce una señal con forma de pico hormonal (pico de LH) que al actuar sobre el ovario produce al menos cuatro efectos:

1. Libera al óvulo de su encierro en las células de la pared folicular y de esa manera el óvulo y las células que lo rodean quedan suspendidos en el fluido folicular.

2. Desencadena una cascada de eventos químicos y celulares que terminan por romper la pared folicular, permitiendo la liberación del óvulo. Este evento tiene lugar entre 24 y 48 horas después del pico de LH.

3. Provoca cambios en las células foliculares, las que disminuyen su producción de estrógeno e inician la producción de $\mathrm{P}$ que, como se dijo anteriormente, induce modificaciones en el cuello uterino, impidiendo la entrada de nuevos espermatozoides, y completa la maduración del endometrio preparándolo para la implantación embrionaria.

4. Induce cambios somáticos y cromosómicos en el óvulo que son necesarios para que, al encontrarse con los espermatozoides, éstos puedan penetrarlo e iniciar la fecundación. Si los óvulos no se exponen al pico de LH y a los cambios hormonales desencadenados posteriormente, no será fecundable o la fecundación será defectuosa.

Es necesario considerar que al referirse a la ovulación, algunos investigadores utilizan exclusivamente el marcador hormonal, es decir la documentación del pico de LH en la sangre u orina; otros utilizan la documentación ecográfica de que hubo ruptura folicular, otros requieren de ambos. En el estudio de Marions y cols. (2002) el tratamiento con LNG dos días antes del pico de LH suprimió el pico en 5 de 5 casos, pero en este estudio no se determinó si hubo o no ruptura folicular. Las biopsias de endometrio en estos casos demostraron alteraciones propias de una insuficiencia en la producción de $\mathrm{P}$ que es esperable al alterar los picos de LH y la consiguiente producción de P. Desde otra perspectiva, pero coherente 
con lo anterior, el trabajo de Durand y cols. (2001), en que se detectó ruptura folicular por ecografía, el tratamiento dado dos, tres y cuatro días antes del pico de LH fue seguido de ruptura folicular y niveles significativamente disminuidos de progesterona en la fase lútea. Sin duda, los efectos del LNG sobre la ovulación parecen depender de la anticipación con que se administra y los tiempos relativos al inicio del pico de LH. Continuando con su línea de investigación, Marions y cols. (2004) intentaron aclarar las dudas generadas en su estudio previo. Para ello, administraron el LNG dos días antes de la ovulación detectada mediante la medición del pico de LH pero, a diferencia de su estudio anterior, siguieron mediante ultrasonografía los ovarios de las mujeres por varios días después del pico de LH. En este estudio se demostró que al postergar o inhibir el pico de LH, en mujeres expuestas al LNG, el folículo dominante o detuvo su desarrollo o simplemente continuó creciendo sin romperse. De esta manera demostraron que en esas condiciones no se liberaba el óvulo del ovario. Por último, Croxatto y cols. (en prensa), usando un elegante diseño metodológico de doble ciego cruzado, randomizado entre LNG y placebo, reafirman los hallazgos anteriores en el sentido de que el LNG aportado antes de la ovulación inhibe, retrasa o altera severamente la ovulación (pico de LH y/o ruptura folicular), lo que se traduce en ausencia de un óvulo fecundable en la trompa de Falopio.

\subsection{LNG en el período posovulatorio}

La polémica que se ha generado por el uso del $\mathrm{AE}$ en este período obedece a la creencia de que si ya existe un óvulo fecundado, el AE puede impedir su desarrollo antes de llegar al útero y/o alterar de tal manera el endometrio, que impida la implantación de un embrión que en ausencia del fármaco habría continuado su desarrollo.

\section{AE y desarrollo embrionario}

No existen estudios in vitro que evalúen el efecto del LNG en el desarrollo preimplantación de embriones humanos, sin embargo dos hechos hacen suponer que no afecta el devenir del embrión.

1. Se sabe que el LNG, a diferencia de las antiprogestinas, no produce aborto o interrupción de un embarazo clínico cuando el embrión ya implantado se expone al LNG.

2. Considerando que la mayoría de los embarazos ocurren cuando el AE es usado después de las 72 horas del coito, y que habitualmente 
esto coincide con fases posovulatorias, es razonable concluir que en la mayoría de los embarazos con $\mathrm{AE}$ el embrión se ha expuesto al LNG durante su fase preimplantacional. No existen evidencias de que los embarazos que se producen como falla del método evolucionen en forma diferente de los embarazos en el grupo control que no estuvieron expuestos al LNG.

\section{$L N G$ y receptividad endometrial}

Como se dijo anteriormente, es poco razonable asumir que el uso posovulatorio de una progestina afecte a un órgano que lo que necesita es su principio activo, la progesterona. El primer estudio que examinó histología endometrial en el período de la implantación, con LNG administrado en los diferentes tiempos después de la ovulación (Landgren, 1989), demostró que el LNG no afecta a la histología endometrial. Existen pocos estudios con adecuados grupos controles que examinen el endometrio expuesto a LNG después de la ovulación en las concentraciones usadas como AE, es decir, dos dosis de 0,75 mg de LNG separados por 12 horas. En el estudio de Marions y cols. (2002) se examinó la biopsia de endometrio tomada en el período de receptividad endometrial en tres mujeres que recibieron LNG dos días antes del pico de LH y en cuatro que lo recibieron dos días después del pico de LH. Se analizaron 8 parámetros morfométricos al microscopio de luz, uno morfológico al microscopio electrónico de barrido y 6 parámetros moleculares por inmunohistoquímica. Las diferencias observadas en relación con los ciclos controles de los mismos sujetos son prácticamente inexistentes.

El estudio de Durand y cols. (2001) analiza 24 biopsias de endometrio obtenidas en ciclos en los que se administró LNG después de la ovulación (documentada por ruptura folicular mediante ultrasonido). No se encontró alteración morfológica de las glándulas endometriales, tampoco del estroma o sistema vascular espiralar.

Estos estudios permiten concluir que a la luz del conocimiento actual no hay evidencias que apoyen la idea de que el LNG usado como AE administrado después de la ovulación altere la funcionalidad endometrial e impida la implantación embrionaria. Existen otras observaciones de tejido endometrial obtenido en mujeres a las que se les administró LNG en dosis mucho mayores que las usadas en AE. Las alteraciones encontradas en esos experimentos son expresión de las concentraciones de la droga y su efecto farmacológico en el tejido endometrial. 
Mona Cebus apella

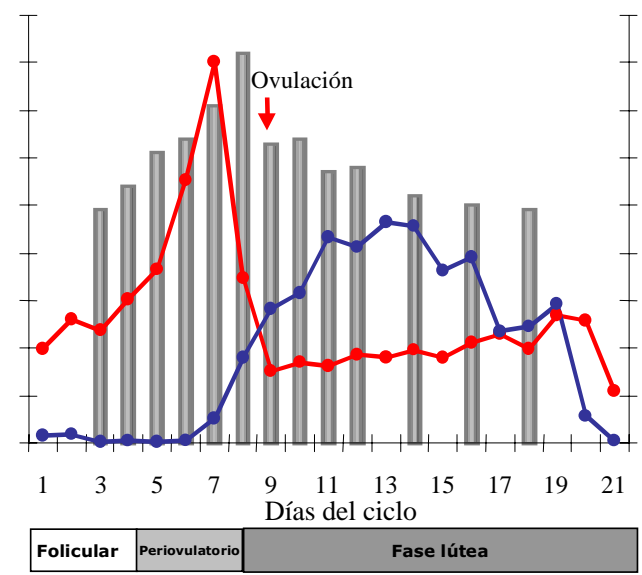

Fases del ciclo reconocidas por citología vaginal

Fuente: Ortiz, M. E. et al.: Human Reproduction, 2004.

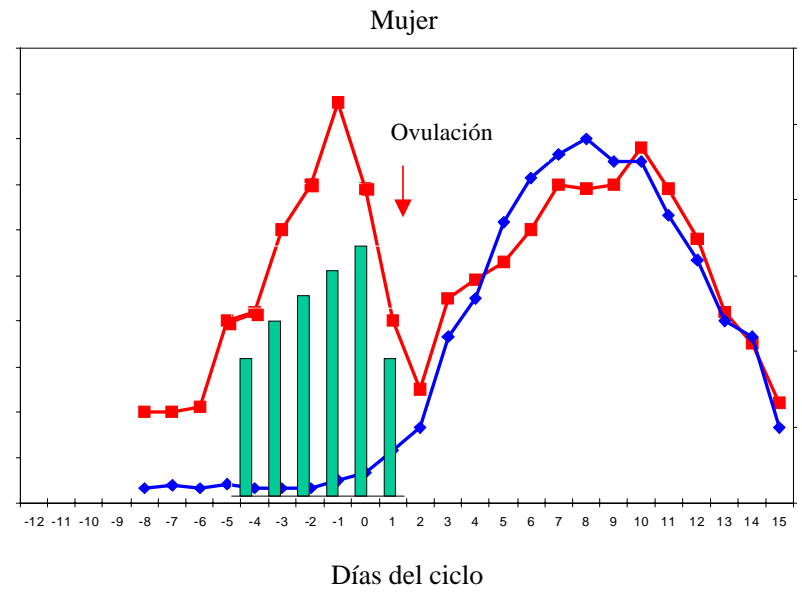

Fuente: Zegers-Hochschild, F.: "The Normal Menstrual Cycle”, 1988.

En relación con el efecto del LNG sobre el embrión preimplantación propiamente tal, no hay pruebas directas en humanos que examinen si existe o no interferencia con la implantación de un óvulo fecundado o un embrión que se ha expuesto al LNG mientras se encuentra navegando a 
través del fluido tubárico o uterino. Por razones de orden ético y de sentido común, nadie generaría embriones humanos con el sólo objeto de someterlos al LNG para ver si se afecta su desarrollo y si se implanta en un endometrio. Es por este motivo que Ortiz y cols. (2004) estudiaron el efecto del LNG administrado en presencia de un óvulo fecundado espontáneamente. El Cebus apella es un mono del Nuevo Mundo cuyo sistema reproductivo comparte muchas características con el de la mujer. En el Gráfico 1 se aprecia la semejanza en la dinámica hormonal, así como en la relación entre los picos hormonales de LH y la ovulación de ambas especies. En este estudio a las monas se les administró LNG en las mismas dosis que se usan como AE en la mujer. Los resultados muestran sin lugar a dudas que el LNG administrado en dosis que fueron capaces de suprimir o postergar la ovulación, no afectó la implantación embrionaria cuando el LNG se administró después de la cópula y de la ovulación, es decir cuando ya existía un óvulo fecundado. El porcentaje de hembras preñadas con y sin LNG fue el mismo.

De esta manera, la información científica sustenta con bastante fuerza que el LNG administrado después de la ovulación no afecta el establecimiento del embarazo y por lo tanto es inefectivo en prevenir que un embrión normalmente constituido se implante en el endometrio.

El conocimiento científico actual relativo al mecanismo de acción del LNG me permite concluir que el LNG $0,75 \mathrm{mg}$ actúa postergando, alterando o inhibiendo la ovulación; alterando o impidiendo la migración espermática y la fecundación. Sin duda, al igual que en la naturaleza, que se expresa en forma espontánea, habrá pérdidas embrionarias espontáneas. Sin embargo, no hay evidencias científicas que puedan sostener que el LNG usado como AE sea responsable de abortos o impida la implantación de embriones normales.

\section{BIBLIOGRAFÍA}

Battaglia, D. E., P. Goodwin, N. A. Klein y M. R. Soules: "Influence of Maternal Age on Meiotic Spindle Assembly in Oocytes from Naturally Cycling Women". En Human Reproduction, 1996, octubre; 11 (10): 2217-22.

Be, C., P. Velásquez y R. Youlton: "Spontaneous Abortion: Cytogenetic Study of 609 Cases". En Revista Médica de Chile, 1997, marzo; 125 (3): 317-22.

Crosby, J. y cols.: "El Corpúsculo Polar. Implicancias Éticas". IFFS 18th World Congress on Fertility and Sterility. 23-28 mayo, 2004. Montreal, Canadá.

Croxatto, H. B., V. Brache, M. Pavez, L. Cochon, M. L. Forcelledo, F. Álvarez, R. Massai, A. Faundes y A. M. Salvatierra: "Pituitary-Ovarian Function Following the Standard Levonorgestrel Emergency Contraceptive Dose or a Single 0.75mg Dose Given on the Days Preceding Ovulation" (en prensa). 
Durand, M., M. Cravioto, E. Raymond, O. Durán-Sánchez, M. Cruz-Hinojosa, A. CastellRodríguez, R. Schiavon y F. Larrea: "On the Mechanisms of Action of Short-Term Levonorgestrel Administration in Emergency Contraception". En Contraception, 2001, 64: 227-234.

Espinos, J., J. Rodríguez-Espinosa, R. Senosiain, M. Aura, C. Vanrell, M. Gispert, C. Vega y J. Calaf: "The Role of Matching Menstrual Data with Hormonal Measurements in Evaluating Effectiveness of Postcoital Contraception”. En Contraception, 1999, 60: 243-247.

Gianaroli, L., M. C. Magli, A. P. Ferraretti, et al.: “Aneuplody Detection in ART". 10 $0^{\text {th }}$ International Congress on Prenatal Diagnosis and Therapy, Barcelona, 19-21, junio, 2000.

Kesseru, E., A. Larrañaga y J. Parada: "Post Coital Contraception with DL-Norgestrel". En Contraception, 1973, 7: 367-79.

Landgren, B. M., E. Johannisson, A-R. Aedo, A. Kumar y S. Yong-en: "The Effect of Levonorgestrel Administered in Large Doses at Different Stages of the Cycle on Ovarian Function and Endometrial Morphology". En Contraception, 1989, 39: 275-89.

Marions, L., K. Gemzell, K. Hultenby, I. Lindell, X. Sun, B. Stabi y M. Bygdeman: "Emergency Contraception with Levonorgestrel and Mifepristone: Mechanism of Action". En American Journal of Obstetrics and Gynecology, 2002, 100 (1): 65-71.

Marions, L., S. Cekan, M. Rygdeman y K. Gemzell-Danielsson: "Effect of Emergency Contraception with Levonorgestrel or Mifepristone on Ovarian Function". En Contraception, 2004, 69: 73-377.

Munné, S., S. Sepúlveda, J. Balmaceda, E. Fernández, C. Fabres, A. Mackenna, T. López, J. A. Crosby y F. Zegers-Hochschild: "Selection of the Most Common Chromosome Abnormalities in Oocytes Prior to ICSI”. En Prenat Diagn, 2000, julio; 20 (7): 582-6.

Ortiz, M. E., R. E. Ortiz, M. A. Fuentes, V. H. Parraguez y H. B. Croxatto: "Post-Coital Administration of Levonorgestrel Does Not Interfere With Post-Fertilization Events in the New-World Monkey Cebus apella". En Human Reproduction, avance publicado en abril 22, 2004.

Senosiain, R., A. Matas, C. Vanrell, S. Peon, I. Mazzanti, M. Gou, M. González, J. Espinos, L. Bassas y J. Calaf: “¿Cuál es el Grado de Exposición Seminal en las Parejas que Solicitan Contracepción Postcoital?" Resúmenes, Cuarto Congreso de la Société Européenne de Gynécologie, Barcelona 25-27 octubre, 2001.

Von Hertzen, H., G. Piaggio, J. Ding, J. Chen, S. Song, G. Bártfai, E. Ng, K. GemzellDanielsson, A. Oyunbileg, S. Wu, W. Cheng, F. Lüdicke, A. Pretnar-Darovec, R. Kirkman, S. Mittal, A. Khomassuridze, D. Apter y A. Peregoudov (para el WHO Research Group on Post-ovulatory Methods of Fertility Regulation): "Low Dose Mifepristone and Two Regimens of Levonorgestrel for Emergency Contraception: A WHO Multicentre Randomized Trial”. En The Lancet, 2002, 360: 1803-1810.

Wilcox, A., C. Weinberg y D. Baird: "Timing of Sexual Intercourse in Relation to Ovulation". En The New England Journal of Medicine, 1995, 333 (23): 1517-1521.

Wilcox, A., C. Weinberg y D. Baird: "Post-Ovulatory Ageing of the Human Oocyte and Embryo Failure". En Human Reproduction, 1998, 13 (2): 394-397.

Zegers-Hochschild, F.: "The Normal Menstrual Cycle". En P. J. Rowe y E. M. Vikhlyaeva (eds.), Diagnosis and Treatment of Infertility. World Health Organization, Hans Huber Publishers, 1988; 137-146.

Zegers-Hochschild, F., C. Fabres y E. Altieri: “Aborto Sub Clínico y Clínico en Fertilización Asistida”. En Revista Latinoamericana de Esterilidad y Fertilidad, 1992, Vol. 6 (3): 107-110. 